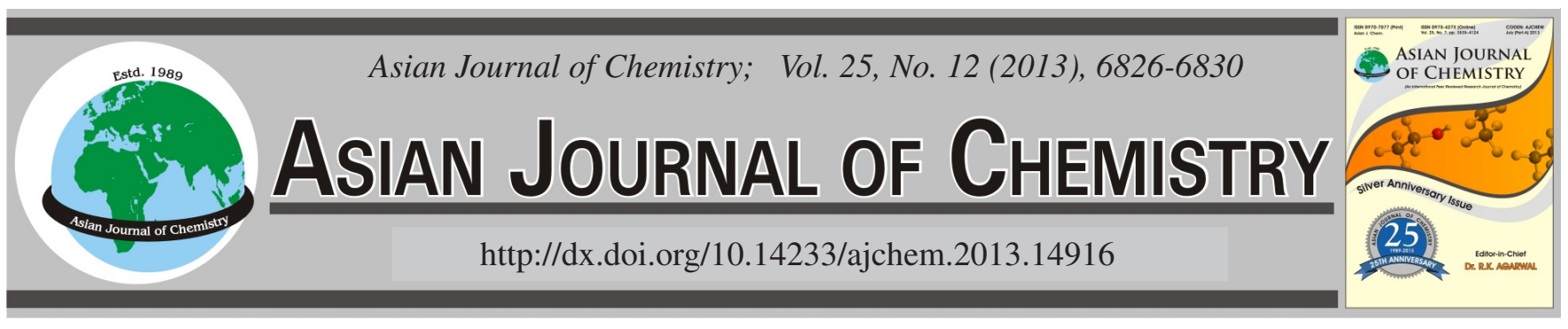

\title{
Investigation of Denitrifying Phosphorus Removal Organisms in a Two-Sludge Denitrifying Phosphorus Removal Process
}

\author{
Haiming Zou ${ }^{1,2}, \mathrm{XIWU}_{\mathrm{Lu}}{ }^{1, *}$ and JiNG SHI ${ }^{1}$
}

${ }^{1}$ School of Energy and Environment, Southeast University, Nanjing 210096, P.R. China

${ }^{2}$ Department of Resource and Environment, Anhui Science and Technology University, Fengyang 233100, P.R. China

*Corresponding author: Tel/Fax: +86 25 83794171; E-mail: xiwulu@ seu.edu.cn

(Received: 4 December 2012;

Accepted: 5 June 2013)

AJC-13581

\begin{abstract}
Denitrifying phosphorus removal organisms in activated sludge samples from a two-sludge denitrifying phosphorus removal process for enhanced biological phosphate removal were investigated by fluorescence in situ hybridization, scanning electron micrograph and energy dispersive spectrometry. The fluorescence in situ hybridization showed that Candidatus accumulibacter phosphatis was dominant in the two-sludge denitrifying phosphorus removal process. By scanning electron micrograph, the morphology of denitrifying phosphorus removal organisms seemed to belong to length streptococcus and its sizes were significantly smaller in anaerobic environment than in anoxic environment. By energy dispersive spectrometry, the phosphorus content of activated sludge collected in this process studied here was $12.3 \%$ of dry weight significantly higher than $3 \%$ in traditional biological phosphorus removal process.
\end{abstract}

Key Words: Denitrifying phosphorus removal organisms, Two-sludge, Fluorescence in situ hybridization.

\section{INTRODUCTION}

Phosphorus is one of the nutrients can causing eutrophication of lakes, inland seas and other natural waters, which threaten the safety of drinking water systems and ecological risk. Removal of phosphorus from wastewater is therefore important for preventing eutrophication. Nowadays, enhanced biological phosphorus removal (EBPR) has been broadly applied in wastewater containing phosphorus treatment because of its lower cost and more environmental friendly technology compared to chemical precipitation or adsorption ${ }^{1}$. Different from chemical or physical treatment methods, EBPR, like other biological wastewater treatment systems, depends on the metabolism of microbial communities to remove phosphorus, possibly removing simultaneously organic or inorganic pollutants. Moreover, EBPR systems are difficult to control and deteriorate in the performance of phosphorus removal ${ }^{2}$. So, a better understanding of microbial communities in EBPR system can give important guidance in design or reconstruction and stable operation of EBPR process ${ }^{3-5}$. Many molecular techniques used for microbial community analysis have been rapidly developed during the past decade, including polymerase chain reaction-denaturing gradient gel electrophoresis (PCRDGGE $)^{6,7}$, fluorescence in situ hybridization $(\mathrm{FISH})^{8-10}$, terminal restriction fragment length polymorphism (T-RFLP) ${ }^{11}$ and clone library ${ }^{12}$.
Compared with traditional microbial identification technology (culture-dependent and isolation), molecular biological techniques can detect various microorganisms including cultured bacteria and uncultured bacteria. These have been successfully used in investigating the diversity and abundance of microorganisms within various wastewater treatment processes. Cui et al. ${ }^{13}$ assessed the microbial communities of three municipal wastewater treatment plants (MWWTPs), including Harbin Taiping, Mudanjiang and Jiamusi MWWTPs located in China, by using PCR-DGGE and reported the dominant microorganisms mainly belonging to Proteobacteria and Bacteroidetes. Contrast to PCR-DGGE, needing destroy bacteria cells for extracting DNA, FISH can keep the original physicochemical condition of monitored samples. So, FISH can be often used to identify microbial communities and detect or quantify target microorganisms ${ }^{14}$. Lu et al. ${ }^{15}$ detected the change of Candidatus accumulibacter Phosphatis, called Accumulibacter for short, considered as a polyphosphate accumulating organism in an EBPR system by using FISH. Kim et al. ${ }^{16}$ also using the FISH technology, investigated the population structure of Accumulibacter in EBPR sludge obtained from two fine-scale sequencing batch reactors (SBRs). However, the diversity and abundance of microorganisms enriched in two-sludge denitrifying phosphorus removal process (proposed in this study, described detail later) have been rarely studied in available literatures. 
In this study, we investigated, using FISH, the diversity and abundance of denitrifying phosphorus removal organisms (DPB), functioning as phosphorus removal bacteria, in two-sludge denitrifying phosphorus removal process, stably running for three years. Moreover, the microbial morphology was assessed by scanning electron micrograph (SEM) and the phosphorus content of sludge samples was analyzed by energy dispersive spectrometry (EDS). To the best of our knowledge, EDS was the first applied in exploring the elements content of activated sludge. Experimental results obtained here would assist in optimization of process parameters, improving design or construction of pilot pant.

\section{EXPERIMENTAL}

Process operation and sludge samples: A lab-scale twosludge denitrifying phosphorus removal process (hereafter referred to as two-sludge DPR process, Fig. 1) investigated in this study was established in 2008 , have been stably running for 3 years. Compared with the conventional wastewater treatment processes, such as sequencing batch reactor (SBR) and anaerobic-anoxic-oxic $\left(\mathrm{A}^{2} \mathrm{O}\right)$ process, the two-sludge DPR process studied here has a great advantage to separate the two parts of activated sludge: nitrification and denitrifying phosphorus removal. This process successfully solves the contradiction of sludge retention time (SRT) between nitrifying bacteria and denitrifying phosphorus removal bacteria, which has applied for a patent (No. 201110431802.4) in China. During the two-sludge DPR process, phosphorus is released anaerobically through the hydrolysis of poly-phosphorus accumulated within intracellular of bacteria, accompanied by the volatile fatty acids (VFA) stored mostly as poly- $\beta$-hydroxybutyrate (PHB) and the degradation of glycogen. And then, a higher amount of phosphorus, in excess of normal metabolic requirements of DPB, is taken up when nitrate or nitrite is supplied instead of oxygen (provided in traditional biological phosphorus removal processes), which is combined with the growth of biomass and the regeneration of glycogen.

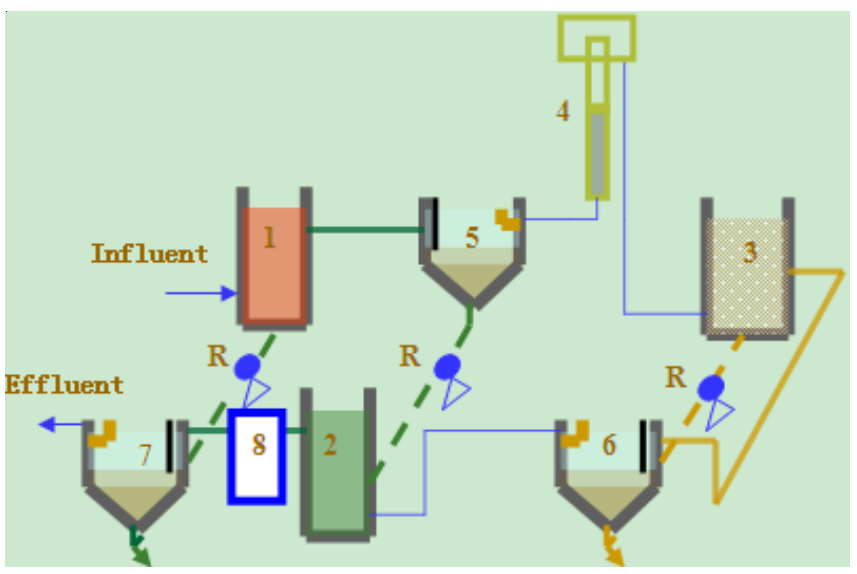

Fig. 1. Denitrifying phosphorus removal in two-sludge and induced crystallization process; 1-anaerobic tank; 2-anoxic tank; 3nitrification tank; 4-induced crystallization column; 5, 6, 7-settling tank; 8-air stripping tank; R-sludge return ratio

Each tank of the two-sludge DPB process was made of poly(vinyl chloride) (PVC), having an effective column of $75 \mathrm{~L}$ in anaerobic tank, $75 \mathrm{~L}$ in anoxic rank, $120 \mathrm{~L}$ in nitrification tank, $86 \mathrm{~L}$ in air stripping tank, $118 \mathrm{~L}$ in induced crystallization column and $72 \mathrm{~L}$ in settling tank. The influent flow rate was pumped at $15 \mathrm{~L} / \mathrm{h}$, with a peristaltic pump. The characteristics of feed were as follows: the COD concentration range of 152$237 \mathrm{mg} / \mathrm{L}, \mathrm{NH}_{4}{ }^{+}-\mathrm{N}$ of $23.4-49.8 \mathrm{mg} / \mathrm{L}$, total nitrogen of 31.3$50.5 \mathrm{mg} / \mathrm{L}$ and total phosphorus 3.92-7.68 mg/L. Correspondingly, the characteristics of final effluent were COD of 10.3-16.1 $\mathrm{mg} / \mathrm{L}$, total nitrogen $9.0-14.5 \mathrm{mg} / \mathrm{L}$ and total phosphorus 0.17 $0.33 \mathrm{mg} / \mathrm{L}$ throughout the 3 years running, meeting the Chinese sewage effluent discharge standards (GB 8978-1996) 1 standard.

Sludge samples for FISH, SEM and EDS were taken from the anaerobic tank included the two-sludge DPB process proposed in this study. For comparison, sludge inoculated to the two-sludge DPB process (namely before cultivation) from an aeration tank of WWTP, Nanjing, China was collected for microbial analysis as well.

\section{Fluorescent in situ hybridization}

Samples pretreatment: Sludge samples were dispersed by ultrasonic for $10 \mathrm{~min}$. And then samples were centrifuged twice at $10000 \mathrm{rpm}$ for $5 \mathrm{~min}$ to remove the impurities, supernatant was discarded and precipitate was washed twice in 0.01 M phosphate buffered saline (PBS) solution.

Samples fixation: Samples treated as mentioned above were fixed with a $4 \%$ paraformaldehyde-PBS solution for $3 \mathrm{~h}$ at $4{ }^{\circ} \mathrm{C}$. Fixed sludge samples were centrifuged (10000 rpm for $5 \mathrm{~min}$ ) and washed twice in $0.01 \mathrm{M} \mathrm{PBS}$ and then resuspended in a PBS-ethanol solution (1:1, vol/vol), being stored at $-20{ }^{\circ} \mathrm{C}$.

Fixed samples dehydration: Before dehydration, the fixed sludge samples were firstly homogenized using a homogenizer for 3 times at $30 \mathrm{~s}$ each time. Homogenized samples were spotted onto slides treated in 3-aminopropyltriethoxysilane (APES) -acetone (1:50, v/v) and then dried for $0.5 \mathrm{~h}$ at $40^{\circ} \mathrm{C}$. Dried slides containing sludge samples were dehydrated for $3 \mathrm{~min}$ in 50, 80 and $100 \%$ (v/v) ethanol and allowed to air-dry and then stored in a desiccator prior to hybridization within FISH experiments.

Hybridization: Hybridization of the treated sludge samples were performed for $2.5 \mathrm{~h}$ at $46^{\circ} \mathrm{C}$ in a $1 \mu \mathrm{L}$ probe $(50$ $\mathrm{ng} / \mu \mathrm{L}$, Table-1) and a $9 \mu \mathrm{L}$ hybridization buffer solution ( $\mathrm{pH}$ 7.2), including $0.9 \mathrm{M} \mathrm{NaCl}, 20 \mathrm{mM}$ Tris- $\mathrm{HCl}, 0.01 \%$ sodium dodecyl sulfate (SDS) and $20 \%$ formamide for EUB338mix, or $35 \%$ for PAOmix.

Washing samples: When fluorescent in situ hybridization for sludge samples completed, a stringent washing step was performed for 4 times at 5 min each time in a washing buffer solution $\left(48^{\circ} \mathrm{C}, \mathrm{pH} 7.2\right)$, including $20 \mathrm{mM}$ Tris- $\mathrm{HCl}, 5 \mathrm{mM}$ EDTA, $0.01 \%$ SDS, $50 \mathrm{mM} \mathrm{NaCl}$. And then these slides attached sludge samples were washed by double distilled water and immediately dried at $30^{\circ} \mathrm{C}$ for $2 \mathrm{~h}$.

Samples observation: The treated slides as mentioned above were observed with a fluorescence microscope (FSX100, Olympus, Japan) with a biological imaging navigator system.

Scanning electron micrograph: Sludge samples collected from anaerobic, anoxic and nitrification tank were fixed in $2.5 \%$ glutaric dialdehyde at $4{ }^{\circ} \mathrm{C}$ for $24 \mathrm{~h}$ and then rinsed 
3 times (15 min. for each time) with a $0.1 \mathrm{M}$ phosphate buffer (PB), $\mathrm{pH}$ 7. For better preservation of cell structure within microorganisms, treated sludge samples were fixed again in $0.1 \%$ acetic acid for $2 \mathrm{~h}$ and then rinsed using the same method mentioned above. Fixed samples were dehydrated in an ethanol series, 50, 70, 80, 90, $100 \%$ ethanol (v/v) for 15 min each. Ethanol in dehydrated samples was displaced by $1: 1(\mathrm{v} / \mathrm{v})$ of ethanol to isoamyl acetate for $0.5 \mathrm{~h}$ with slightly shaking and then by $100 \%$ isoamyl acetate for $0.5 \mathrm{~h}$. These treated samples dried by the $\mathrm{CO}_{2}$ critical point drying. The treated sludge samples were observed after spray-gold treatment, using a JSM-6360LV (Japan) SEM.

X-ray energy spectrum analysis: Phosphorus content in the treated sludge samples (in scanning electron micrograph) was investigated by a GENESIS 2000 XMS (USA) EDS.

\section{RESULTS AND DISCUSSION}

In situ hybridization for DPB: The EUBmix labeled with FITC was used to target all bacterial community and the PAOmix labeled with CY3 was used to target Accumulibacter, as shown in Table-1. FISH analysis was conducted here to detect the variation of Accumulibacter within anoxic tank by means of in situ hybridization with 16SrRNA-targeted oligonucleotide probes enabling the identification and monitoring of the specific strains in the microbial community (Fig. 2). Fig. 2(a) and (b), representing uncultivated sludge samples and cultivated sludge samples respectively, show FISH images of Accumulibacter, shown in yellow.

Accumulibacter belonging to Rhodocyclus-related within Betaproteobacteria has been demonstrated as an important phosphorus removal microorganism in EBPR systems as reviewed by He and McMahon ${ }^{17}$. The abundance of Accumulibacter in the two-sludge DPB process proposed here was detected using PAOmix probe (Table-1) and results were shown in Fig. 2(a) and (b). Accumulibacter was low abundance in uncultivated sludge [Fig. 2(a)], collected from WWTP, Nanjing, China, further demonstrating the fact $^{18}$ that lower phosphorus removal efficiency occurs in conventional wastewater treatment systems compared with the EBPR process. In contrast to this result, Accumulibacter was good growth in the two-sludge DPB system during the whole running period over three years. FISH image [Fig.2 (b)] shows Accumulibacter, shown in yellow, was a considerable proportion in anoxic tank. This find is well consistent with the good phosphorus removal performance observed in this system in our previous experiments ${ }^{19}$. The results of elements analysis by EDS that the content of phosphorus contained in anoxic sludge was significantly higher than in traditional activated sludge, as discussed in the energy dispersive spectrometry for DPB in this paper.

The low abundance of Accumulibacter in the traditional wastewater treatment system could be because of the existence of the contradiction of sludge age between nitrification and phosphorus removal performance ${ }^{20}$. However, in the two-sludge DPB system studied here, sludge functioning as nitrification and one functioning as denitrifying phosphorus removal were cultivated separately in two independent tanks, which could completely resolve the contradiction of sludge age mentioned above, can providing a more suitable environment for the growth of Accumulibacter. To get a better understanding of Accumulibacter in the two-sludge DPB process, the observation of microbial morphologies should be conducted and characterized.

Scanning electron micrograph for DPB: The morphologies of DPB developed under the conditions of anaerobic and anoxic over the three year time period, as obtained by SEM are illustrated in Fig. 3. For comparison, the morphologies of microbes in inoculated sludge $(\mathrm{CK})$ collected from the aerobic pond of WWTP, Nanjing, China, were investigated as well. SEM micrographs of sludge sampled from two reactors

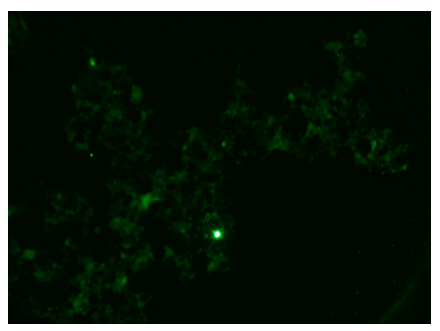

(a)

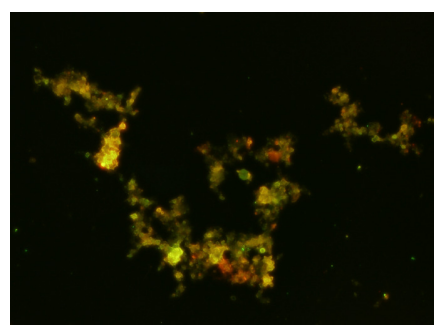

(b)

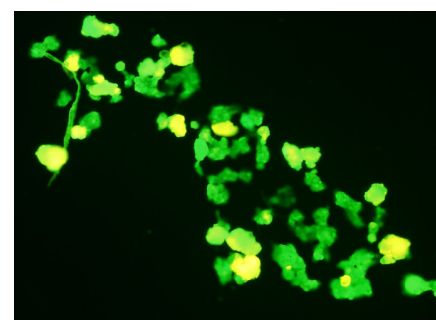

(c)

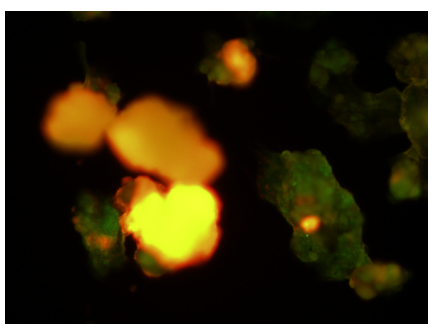

(d)

Fig. 2. FISH images from anoxic tank and nitrification tank before or after cultivated sludge; a: uncultivated nitrification sludge; b, c, d: cultivated sludge, representing for FISH images amplified by 100 times, 300times, 600times; Accumulibacter are shown in yellow combined green and red and all other bacteria are shown in green. To the best of our knowledge, FISH image amplified by 600 times for DPB was firstly reported, showing clearly polyphosphate granules

\begin{tabular}{|c|c|c|c|c|c|}
\hline \multicolumn{6}{|c|}{$\begin{array}{c}\text { TABLE-1 } \\
\text { FISH PROBES USED IN THIS STUDY }\end{array}$} \\
\hline Probe mix & Probe & Sequence (5'-3') & rRNA & Target & Dye 5 , \\
\hline \multirow{3}{*}{ EUBmix } & EUB338 & GCTGCCTCCCGTAGGAGT & $16 \mathrm{~S}$ & $338-355$ & FITC \\
\hline & EUB338II & GCAGCCACCCGTAGGTGT & $16 \mathrm{~S}$ & $338-355$ & FITC \\
\hline & EUB338III & GCTGCCACCCGTAGGTGT & $16 \mathrm{~S}$ & $338-366$ & FITC \\
\hline \multirow{3}{*}{ PAOmix } & PAO651 & CCCTCTGCCAAACTCCAG & $16 \mathrm{~S}$ & $651-668$ & Cy3 \\
\hline & PAO462 & CCGTCATCTACWCAGGGTATTAAC & $16 S$ & $462-485$ & Су 3 \\
\hline & PAO846 & GTTAGCTACGGCACTAAAAGG & $16 S$ & $846-866$ & Су 3 \\
\hline
\end{tabular}

EUBmix used to target all bacteria; PAOmix used to target Accumulibacter. 


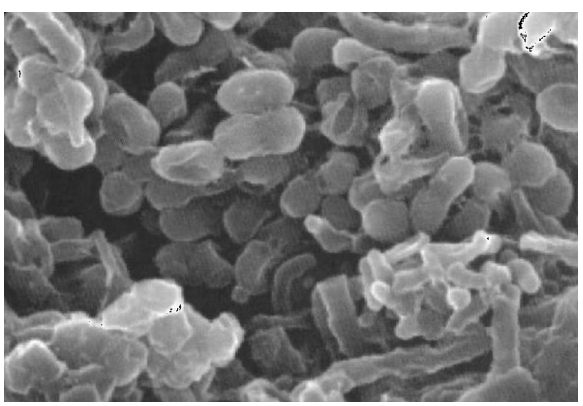

(a)

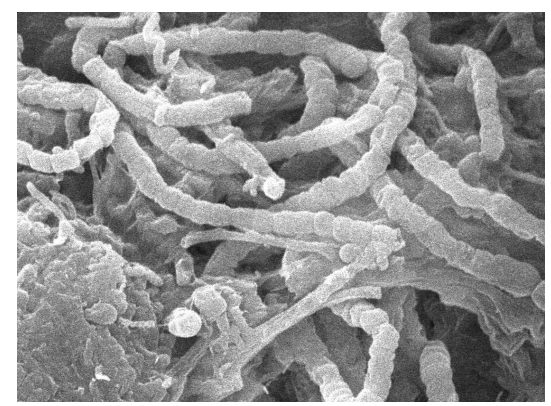

(b)

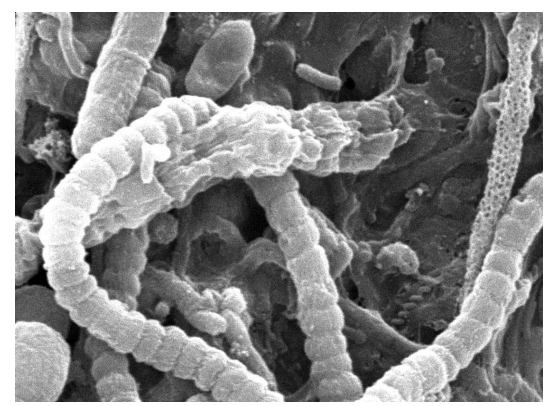

(c)

Fig. 3. Image of electronic scanning microscopy for sludge before or after cultivated within anaerobic pond, anoxic pond. a: before cultivated sludge; b: anaerobic tank; c: anoxic tank. All images obtained here were amplified by 5000 times

and $\mathrm{CK}$ showed different microbial morphologies in each sample. This suggests that different microbial morphologies could correspond to different strains enriched in each reactor that possess different metabolic performances and perhaps display different affinities for substrates, nitrogen, phosphorus or oxygen, etc.

Before the acclimatization process, the SEM image indicated that inoculated sludge seemed to consist of coccus morphology and coccus-rod morphology [Fig.3 (a)], diameters ranging from 1.2 to $1.6 \mu \mathrm{m}$, with one or two individual combination. According to the microbial maps, these microbes observed in inoculated sludge could be sphaerotilus and corynebacterium, functioning as hydrolysis or nitrifying bacteria. These are generally consistent with a recent study, where proteobacteria was found to the dominant phylum in aeration activated sludge sampled from 12 municipal WWPTs with different treatment processes in China.

Microbial morphology analysis of anaerobic and anoxic sludge by SEM, [Fig. 3(b), (c)], suggested that the primary DPB cultivated in these two tanks during the three years period seemed to be length streptococcus, belonging to streptococcaceae, lactobacillales, bacilli. However, the sizes of DPB in anaerobic and anoxic conditions were apparently different. Its sizes in anaerobic environment were averaged $0.85 \mu \mathrm{m}$ smaller than in anoxic environment (averaged $1.26 \mu \mathrm{m}$ ), indicating the higher uptake of DPB to poly-phosphorus than to volatile fatty acid.
Energy dispersive spectrometry for DPB: Energy dispersive spectrometry (EDS) is a process, during which qualitative and quantitative analysis of a sample would be done by excitation of atoms, leading to the production of X-ray, the energies of which differ for different elements. In denitrifying phosphorus removal process, high phosphorus removal efficiency can be achieved by withdrawing the excess sludge with high phosphorus content ${ }^{21}$. The differences of phosphorus content between anaerobic sludge and anoxic sludge in twosludge DPB process were investigated by EDS in this study, (Fig. 4).

There was a significant difference in the phosphorus content of sludge between anaerobic and anoxic in the twosludge DPB process here (Fig. 4). The excited atoms counts of phosphorus element in the anaerobic sludge were $0.55 \mathrm{k}$, while were $1.18 \mathrm{k}$ in the anoxic sludge, suggesting that the difference of phosphorus content in the denitrifying phosphorus removal sludge between anaerobic and anoxic should be due to the different performances of DPB in different conditions, including that phosphorus was released anaerobically and taken up anoxically. The phosphorus content of excess sludge in this study, namely anoxic sludge, was $12.3 \%$ of dry weight significantly higher than in traditional biological phosphorus removal process $(3 \%)^{22}$, suggesting that two independent sludge cultivation systems proposed here, separating the nitrifying sludge from denitrifying phosphorus removal

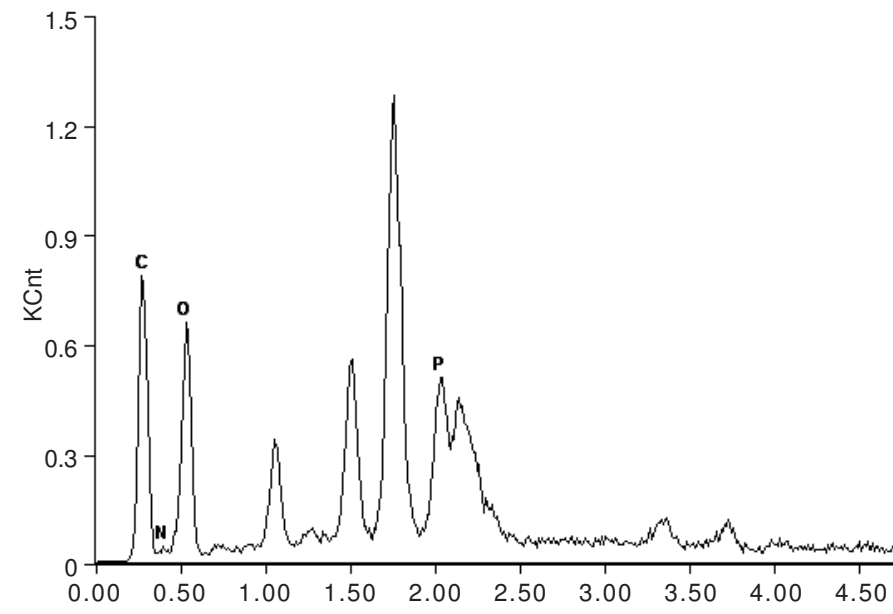

(a) Anaerobic sludge

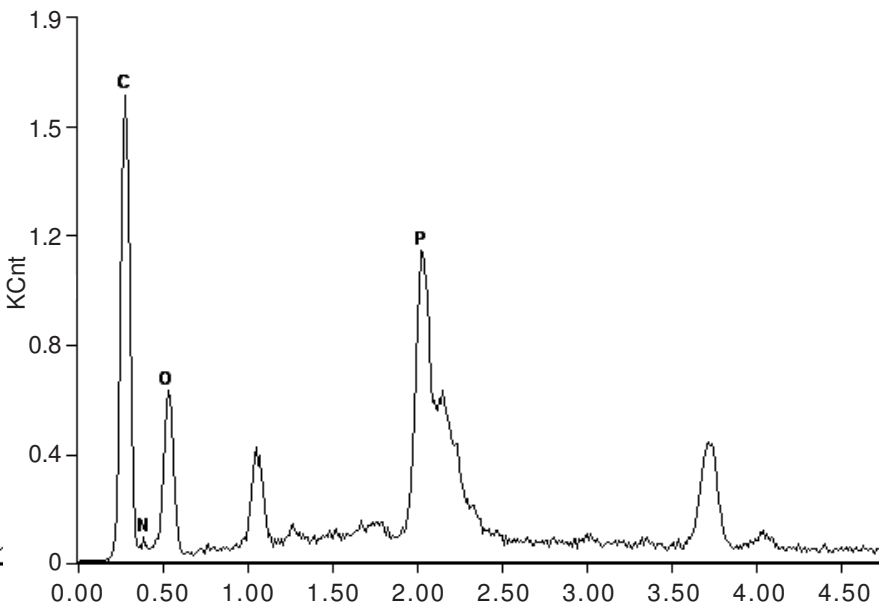

(b) Anoxic sludge

Fig. 4. Analytical X-ray spectrum of anaerobic sludge and anoxic sludge in two-sludge DPB process; The spectrum was given with energies of these X-rays $(\mathrm{KeV})$ as abscissa and specific counts of each specific energy as vertical coordinate (KCnt) 
sludge, could effectively enhance the phosphorus removal efficiency, which are consistent with the results of chemical analysis ${ }^{19}$. Further research is needed to determine the relative distributions of phosphorus contained in and intracellular structures and extracellular polysaccharides.

\section{Conclusion}

In this paper, fluorescence in situ hybridization (FISH), scanning electron micrograph (SEM) and energy dispersive spectrometry (EDS) were applied for the in situ identification of denitrifying phosphorus removal organisms (DPB), microbial morphology observation of DPB and phosphate content analysis of DPB in two-sludge denitrifying phosphorus removal process. The following conclusions were drawn from these results.

(1) FISH technology was useful to identify the diversity and abundance of DPB in the two-sludge denitrifying phosphorus removal process. Throughout the three years running, Accumulibacter was greatly accumulated in process.

(2) Different microbial morphologies were observed by SEM in different ponds. For inoculated sludge, coccus morphology and coccus-rod morphology of microorganisms were dominant and seemed to be sphaerotilus and corynebacterium, functioning as hydrolysis or nitrifying bacteria. For anaerobic and anoxic sludge, the sizes of DPB in anaerobic environment were smaller than in anoxic environment.

(3) EDS method was rather effective in investigating the phosphorus content of activated sludge. The phosphorus content of excess sludge in this study was $12.3 \%$ of dry weight significantly higher than in traditional biological phosphorus removal process $(3 \%)$.

\section{ACKNOWLEDGEMENTS}

The authors thank M.M. Su for assistance in activated sludge treatment and Dr. Wang for enlightening discussion. This research is supported by grant 2012ZX07101-005 from National Key Technology in Water Pollution Control and Treatment in 12th Five-year Plan of China and grant 51078074 from National Natural Science Foundation of China.

\section{REFERENCES}

1. M. Pijuan, A.M. Saunders, A. Guisasola, J.A. Baeza, C. Casas and L.L. Blackall, Biotechnol. Bioeng., 85, 56 (2004).

2. W.J. Lu, D.H. Yang and Y. Guo, Environ. Pollut. Control, 33, 2709 (2012).

3. D.B. Oerther, F.L. De Los Reyes, M.F. De Los Reyes and L. Raskin, Water Res., 35, 3325 (2001).

4. K.M. DeAngelis, C.H. Wu, H.R. Beller, E.L. Brodie, R. Chakraborty, T.Z. DeSantis, J.L. Fortney, T.C. Hazen, S.R. Osman, M.E. Singer, L.M. Tom and G.L. Andersen, Appl. Environ. Microb., 77, 6313 (2011).

5. M. Hu, X.H. Wang, X.H. Wen and Y. Xia, Bioresour. Technol., 117, 72 (2012).

6. S. He, A.Z. Gu and K.D. McMahon, Water Sci. Technol., 54, 111 (2006).

7. D.R. de Figueiredo, R.V. Ferreira, M. Cerqueira, T.C. de Melo, M.J. Pereira, B.B. Castro and A. Correia, Environ. Monit. Assess., 184, 471 (2012).

8. G. Carvalho, P.C. Lemos, A. Oehmen and M.A.M. Reis, Water Res., 41, 4383 (2007).

9. A. Oehmen, Z.G. Yuan, L.L. Blackall and J. Keller, Biotechnol. Bioeng., 91, 162 (2005).

10. B.-K. Hwang, C.-H. Lee, I.-S. Chang, A. Drews and R. Field, J. Membr. Sci., 419-420, 33 (2012).

11. L. Chen, M.T. Teasdale, M.M. Kaczmarczyk, G.G. Freund and M.J. Miller, J. Microbiol. Meth., 91, 262 (2012).

12. S. Li, L. Sun, H. Wu, W. Liu, Y. Li and X. Wen, J. Appl. Microbiol., 113, 1341 (2012).

13. D. Cui, A. Li, S. Zhang, C.L. Pang, J.X. Yang, J.B. Guo, F. Ma, J.H. Wang and N.Q. Ren, World J. Microbiol. Biotechnol., 28, 2341 (2012).

14. H. Kang, X. Wang and N. Li, J. Harbin Inst. Technol., 3, 13 (2011).

15. H. B. Lu, A. Oehmen, B. Virdis, J, Keller and Z.G. Yuan, Water Res., 40, 3838 (2006).

16. J.M. Kim, H.J. Lee, S.Y. Kim, J.J. Song, W. Park and C.O. Jeon, Appl. Environ. Microb., 76, 3825 (2010).

17. S. He and K.D. McMahon, Microb. Biotechnol., 4, 603 (2011).

18. C.M. Falkentoft, E. Müller, P. Arnz, P. Harremoës, H. Mosbæk, P.A. Wilderer and S. Wuertz, Water Res., 36, 491 (2002).

19. J. Shi, X.W. Lu and Y.F. Wu, CIESC J., 61, 1255 (2010) (in Chinese).

20. L.-F. Shen, R.-P. Chen, F.W. Zhang, J.-T. Yang and Y. Li, Procedia Environ. Sci., 11, 1291 (2011).

21. T. Mino, M.C.M. Van Loosdrecht and J.J. Heijnen, Water Res., 32, 3193 (1998).

22. P.H. Nielsen, A.M. Saunders, A.A. Hansen, P. Larsen and J.L. Nielsen, Curr. Opin. Biotechnol., (2011). 